\title{
Existence of a Bilinear Differential Realization in the Constructions of Tensor Product of Hilbert Spaces
}

\author{
ALEKSEY DANEEV \\ Department of Information Systems and Information Protection \\ Irkutsk State Transport University \\ st. Chertyshevsky, 15, Irkutsk \\ RUSSIA

\begin{abstract}
ANATOLIY LAKEYEV
Department of Dynamics and Control

Institute for System Dynamics and Control Theory

of the Siberian Branch of the Russian Academy of Sciences st.

Lermontova, 134, Irkutsk

RUSSIA

VYACHESLAV RUSANOV

Department of Dynamics and Control

Institute for System Dynamics and Control Theory

of the Siberian Branch of the Russian Academy of Sciences st.

Lermontova, 134, Irkutsk

RUSSIA
\end{abstract}

\begin{abstract}
The paper describes the results of a functional-geometric study of the necessary and sufficient conditions for the existence of a differential realization in the terms of the tensor product of real Hilbert spaces. There are considered continuous infinite-dimensional dynamical system in the class of controlled bilinear nonstationary ordinary differential equations of the second order (including quasi-linear hyperbolic models) in a separable Hilbert space. Therefore the topological and metric conditions for the continuity of the Rayleigh-Ritz operator with the calculation of the fundamental group of its image are analytically substantiated. The results of paper give incentives for generalizations in the qualitative theory of nonlinear structural identification of higher order multi-linear differential models.
\end{abstract}

Key-Words: - tensor analysis, nonlinear system analysis, differential realization, Rayleigh-Ritz operator.

Received: November 8, 2019. Revised: March 20, 2020. Accepted: April 8, 2020. Published: April 25, 2020.

\section{Introduction}

Since the qualitative theory of differential realization studies the problems of the existence of analytical representation for a properly defined continuous dynamical system [1], the present article could have the title: How to determine whether bilinear differential "black box" equation exists. Therefore, from a methodological point of view, bilinear differential realization can be considered as an important step towards constructing a general theory of mathematical modeling of complex dynamic systems within the context of the theory of identification of evolu- tionary equations [2] at the junction of functional analysis $[3,4]$ and the theory of differential equations in infinite-dimensional spaces [5]. Moreover, in the system-theoretical analysis of the continuous weakly structured systems, up to a certain moment, the qualitative results of finite-dimensional realization theory are "mechanically" transferred to the infinite-dimensional case without particular complications. This applies to both stationary and non-stationary differential models of the first order (parabolic equations and systems of diffusion type) in uniformly convex Banach spaces [6] and separable Hilbert spaces $[7,8]$. 
Significant analytical difficulties can be faced when moving to constructing a differential realization with a dynamic order higher than the first [9]. This includes the non-nominal consideration of the structure of hyperbolic models [10], the representations of which cannot dispense with taking into account the nonlinearity of their dynamics, in particular, the bilinear structure of the implementation model [11,12], which is the focus of attention in this article. At the same time, we show that, throwing the analytical bridge between projective geometry and the differential realization of simulated infinite- dimensional dynamic processes, the structure of projectivization of the non-linear functional Rayleigh -Ritz operator [7, 8] and the functional-geometric analysis of its continuity conditions can be conveniently formulated in a language of compact topological varieties of tensor products of Hilbert spaces in terms of the Whitehead CW -complexes [13].This kind of qualitative theory has not yet been fully created and most recently its base did not exist. Over the past decade and a half, the situation has changed significantly [6-12], and it can be assumed that now this base exists (see works [14], [15] where the main studies in this area are summarized).

\section{Problem Formulation}

Further on, $\left(X,\|\|_{X}\right),\left(Y,\|\|_{Y}\right),\left(Z,\|\|_{Z}\right)$ are real separable Hilbert spaces (that is, norms satisfy "the condition of parallelogram" [16, p. 47]); with that, below we use [3, p. 176] the linear isometry (preserving the norm) $E: Y \rightarrow X$ of the spaces $Y$ and $X$. As usual, $L\left(\mathscr{B}^{\prime}, \bar{\delta}^{\prime \prime}\right)$ is the Banach space (with operator norm) of all linear continuous operators for the Banach spaces $\mathscr{W}^{\prime}$ and $\mathscr{W}^{\prime \prime}, \mathscr{L}\left(X^{2}, X\right)$ is the space of all continuous bilinear mappings from the Cartesian square $X \times X$ (with product topology) into the space $X$ (similar to $\mathcal{L}\left(X^{2}, Z\right)$ ).

Let $T$ denote the segment of the number scale $R$ with the Lebesgue measure $\mu$, and $\wp_{\mu}$ denote the $\sigma$-algebra of all $\mu$-measurable subsets out of $T$, the notation of $S \underset{\text { mod } \mu}{\subseteq}$ for $S, Q \in \wp_{\mu}$ means $\mu(S \backslash Q)=0$.

Additionally, we assume that $A C^{1}(T, X)$ is the set of all functions $\varphi: T \rightarrow X$, the first derivative of which is absolutely continuous function (with respect to measure $\mu$ ) on the interval $T$.

If below $(\mathscr{B},\|\cdot\|)$ is some Banach space, then let $\mathrm{L}_{p}(T, \mathscr{B}), p \in[1, \infty)$ denote the Banach space of all classes of $\mu$-equivalence of all Bochner-integrable
[4] mappings $f: T \rightarrow \mathscr{\mathcal { B }}$ with norm $\left(\iint_{T} \mid f(\tau) \|^{p} \mu(d \tau)\right)^{1 / p}$, respectively, let $\mathrm{L}_{\infty}(T, \widetilde{\mathcal{B}})$ denote the Banach space

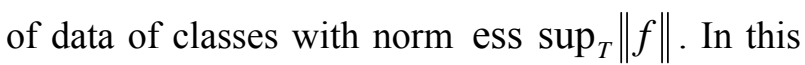
context, we agree that

$$
\begin{gathered}
\mathrm{L}_{2}:=\mathrm{L}_{2}(T, \mathrm{~L}(X, X)) \times \mathrm{L}_{2}(T, \mathrm{~L}(X, X)) \times \mathrm{L}_{2}(T, L(Y, X)) \times \\
\times \mathrm{L}_{2}\left(T, \mathcal{L}\left(X^{2}, X\right)\right) \times \mathrm{L}_{2}\left(T, \mathcal{L}\left(X^{2}, X\right)\right) \times \\
\times \mathrm{L}_{2}\left(T, \mathcal{L}\left(X^{2}, X\right)\right) \times \mathrm{L}_{2}\left(T, \mathcal{L}\left(X^{2}, X\right)\right) \times \mathrm{L}_{2}\left(T, \mathcal{L}\left(X^{2}, X\right)\right), \\
\quad \mathrm{L}^{*}:=L(X, X) \times L(X, X) \times L(Y, X) \times \\
\times \mathcal{L}\left(X^{2}, X\right) \times \mathcal{L}\left(X^{2}, X\right) \times \mathcal{L}\left(X^{2}, X\right) \times \mathcal{L}\left(X^{2}, X\right) \times \mathcal{L}\left(X^{2}, X\right) .
\end{gathered}
$$

Further, we believe that, on the time interval $T$, the behavior $[1,6,15]$ of the studied behavioral system in the form of a nonlinear - and not limited in power-bundle $N$ of controlled nonlinear dynamic processes (controlled trajectory curves) of the "input-output" type is recorded (possibly a posteriori), i.e. formally:

$$
\begin{gathered}
N \subset\left\{(x, u): x \in A C^{\mathrm{l}}(T, X), u \in \mathrm{L}_{2}(T, Y)\right\}, \\
\operatorname{Card} N \leq \exp \aleph_{0},
\end{gathered}
$$

where $(x, u)$ is the pair "trajectory, program control", $\aleph_{0}$ is the alef-zero, exp $\aleph_{0}$ is the continuum; the term "nonlinear bundle" means that for the trajectory curves of this bundle the presence of the superposition principle is not assumed a priori $[17, \mathrm{p}$. 18], when the dependence of the output quantities $x(\cdot)$ on the input actions $u(\cdot)$ is linear.

Next, let the statement function for the second derivative (in the implementation model) of the trajectory $t \mapsto x(t)$ of the form below be given as the inertial-mass characteristic of the simulated system:

$$
\begin{gathered}
\hat{A} \in \mathrm{L}_{\infty}(T, L(X, X)), \\
\mu\{t \in T: \hat{A}(t)=0 \in L(X, X)\}=0 ;
\end{gathered}
$$

taking into account further constructions, we assume that the violation of the condition

$$
\mu\{t \in T: \operatorname{Ker} \hat{A}(t)=0 \in X\}=0
$$

is permissible.

Let us consider the problem: ${ }^{1}$ for the pair $(N, \hat{A})$ defined, determine the necessary and sufficient conditions expressed in terms of a nonlinear bundle of $N$ dynamic processes and the statement function

\footnotetext{
${ }^{1}$ One of the founders of the realization theory, R.E. Kalman, stating that in the general theory of systems the realization problem plays a central role [17, p. 267], formulated the following approach [17, p. 286]: to consider the realization problem as an attempt to guess the equations of motion of a dynamic system by the behavior of its input and output signals or as building a physical model that explains the experimental data.
} 
$\hat{A}$, for the existence of an ordered set of eight statement functions

$$
\left(A_{1}, A_{0}, B, D_{1}, D_{2}, D_{3}, D_{4}, D_{5}\right) \in L_{2},
$$

for which a bilinear differential realization (BDR) of the following form is feasible:

$$
\begin{gathered}
\hat{A} d^{2} x / d t^{2}+A_{1} d x / d t+A_{0} x= \\
=B u+\mathrm{D}_{1}(x, x)+\mathrm{D}_{2}(x, d x d t)+\mathrm{D}_{3}(d x d d t, d x d t)+ \\
+\mathrm{D}_{4}(E(u), x)+\mathbb{D}_{5}(E(u), d x / d t) \quad \forall(x, u) \in N ;
\end{gathered}
$$

equality in (1) is considered as identity in $\mathrm{L}_{1}(T, X)$. If the simulated operators of the BDR-system (1) are supposed to be searched for in the class of stationary operators, then we will construct them in the class of continuous operators, and write

$$
\left(A_{1}, A_{0}, B, D_{1}, D_{2}, D_{3}, D_{4}, D_{5}\right) \in \mathbb{L}^{*} \text {. }
$$

In connection with the indicated mathematical formulation, we note that each area of mathematics, as a rule, contains its major problems, which are so difficult that their complete solution is not even expected, but they stimulate a constant work flow and serve as milestones on the way to progress in this area. In the qualitative theory of differential realization of dynamic systems, such a problem is that of classifying continuous infinite-dimensional behavioristic systems, considered as if they exactly coincided with the solutions of idealized differential models. In the most strong form, it suggests the classification of such systems within the accuracy of the corresponding class of differential realization models, in particular, the class of non-stationary BDRmodels (1), which are substantiated below by Theorems 1,2 (and their corollaries) that allow in the aforementioned classification to significantly approach the ideal combination of functional transparency and geometric visibility.

\section{The Existence of the BDR-Model}

We now describe the analytic scheme on the solvability of the BDR-problem (1). So, let $Z:=X \otimes X$ be an updated tensor product [4, p. 64] of Hilbert spaces $X$ and $X$ with cross-norm \|\|$_{Z}$, defined by the internal product. Moreover, we accept the following notation:

$$
\begin{gathered}
U:=X \times X \times Y \times Z \times Z \times Z \times Z \times Z, \\
\|(;, ; ; \cdot)\|_{U}:=\left(\|\|_{X}^{2}+\|\|_{X}^{2}+\|\|_{Y}^{2}+\|\|\left\|_{Z}^{2}+\right\| \cdot\left\|_{Z}^{2}+\right\|\left\|_{Z}^{2}+\right\|\left\|_{Z}^{2}+\right\| \|_{Z}^{2}\right)^{1 / 2} ; \\
\mathrm{L}_{2}:=\mathrm{L}_{2}(T, L(X, X)) \times \mathrm{L}_{2}(T, L(X, X)) \times \mathrm{L}_{2}(T, L(Y, X)) \times \\
\times \mathrm{L}_{2}(T, L(Z, X)) \times \mathrm{L}_{2}(T, L(Z, X)) \times \mathrm{L}_{2}(T, L(Z, X)) \times \\
\times \mathrm{L}_{2}(T, L(Z, X)) \times \mathrm{L}_{2}(T, L(Z, X)) ;
\end{gathered}
$$

it is clear that functional space $\mathbf{L}_{2}$ (with the topology of the product) is linearly homeomorphic to the Banach space $\mathrm{L}_{2}(T, L(U, X))$.

Let $\pi$ denote the universal bilinear mapping

$$
\pi: X \times X \rightarrow X \otimes X
$$

in the language of categories, morphism $\pi$ defines the tensor product as a universally repelling object $[16$, p. 40]. The universality of the bilinear mapping $\pi$ also consists in the following relations being satisfied:

$$
\begin{gathered}
\pi: X \times X \rightarrow X \otimes X, \\
\left(\mathrm{x}_{1}, \mathrm{x}_{2}\right) \mapsto \pi\left(\mathrm{x}_{1}, \mathrm{x}_{2}\right)=\mathrm{x}_{1} \otimes \mathrm{x}_{2}, \\
\left\|\mathrm{x}_{1} \otimes \mathrm{x}_{2}\right\|_{Z}=\left\|\mathrm{x}_{1}\right\|_{X}\left\|\mathrm{x}_{2}\right\|_{X} ;
\end{gathered}
$$

these relations are important for determining the construction of the functional Rayleigh-Ritz operator (2) in terms of specification of the norm $\|\cdot\|_{U}$.

We further believe that the Cartesian square $X^{2}=X \times X$ is endowed with the norm $\left(\|\cdot\|_{X}^{2}+\|\cdot\|_{X}^{2}\right)^{1 / 2}$. In this formulation, $\pi \in \mathscr{L}\left(X^{2}, Z\right)$ holds. Moreover, taking into account Theorems 2 [3, p. 245] for any bilinear mapping $\mathscr{D} \in \mathcal{L}\left(X^{2}, X\right)$, there exists a linear continuous operator $\mathrm{D} \in L(Z, X)$, such that $\mathscr{D}=\mathrm{D} \circ \pi$. is feasible. In addition, for any pair $(x, u) \in N$ the following inclusions will be performed:

$$
\begin{gathered}
\pi(x, x), \pi\left(x, d x^{\prime} d t\right), \pi(d x d d t, d x d d t) \in \mathrm{L}_{\infty}(T, Z), \\
\pi(E(u), x), \pi(E(u), d x / d t)) \in \mathrm{L}_{2}(T, Z) .
\end{gathered}
$$

These formulations are summarized below by the following statement.

Lemma 1. For any set of statement functions and mapping

$$
\left(A, A_{y}, B, D_{1}, D_{2}, D_{3}, D_{4}, D_{5}\right) \in L_{2}
$$$$
\left(A, A_{3}, B_{1} D_{1}, D_{2}, D_{3}, D_{4}, D_{5}\right) \in L_{2}
$$$$
F: \mathrm{L}_{2}(T, X) \times \mathrm{L}_{2}(T, X) \times \mathrm{L}_{2}(T, Y) \times
$$$$
\times \prod_{i=1}^{5} \mathrm{~L}_{2}\left(T, X^{2}\right)_{i} \rightarrow \mathrm{L}_{1}(T, X),
$$$$
\left(y_{1}, y_{2}, y_{3}, y_{4}, y_{5}, y_{6}, y_{7}, y_{8}\right) \mapsto F\left(y_{1}, y_{2}, y_{3}, y_{4}, y_{5}, y_{6}, y_{7}, y_{8}\right):=
$$$$
=A y_{1}+A y_{2}+B y_{3}+D_{1} y_{4}+D_{2} y_{5}+D_{3} y_{6}+D_{4} y_{7}+D_{5} y_{8} \text {, }
$$

there exists a unique tuple of statement functions

$$
\left(D_{1}, D_{2}, D_{3}, D_{4}, D_{5}, D_{6}, D_{3}, D_{8}\right) \in \mathbf{I}_{2}
$$

and, accordingly, a unique linear mapping

$$
M: \mathrm{L}_{2}(T, U) \rightarrow \mathrm{L}_{1}(T, X),
$$

having an analytical representation written as

$$
\begin{aligned}
& \left(\mathrm{z}_{1}, \mathrm{z}_{2}, \mathrm{z}_{3}, \mathrm{z}_{4}, \mathrm{z}_{5}, \mathrm{z}_{6}, \mathrm{z}_{7}, \mathrm{z}_{8}\right) \mapsto M\left(\mathrm{z}_{1}, \mathrm{z}_{2}, \mathrm{z}_{3}, \mathrm{z}_{4}, \mathrm{z}_{5}, \mathrm{z}_{6}, \mathrm{z}_{7}, \mathrm{z}_{8}\right):= \\
& =D_{1} \mathrm{z}_{1}+D_{2} \mathrm{z}_{2}+D_{3} \mathrm{z}_{3}+D_{4} \mathrm{z}_{4}+D_{5} \mathrm{z}_{5}+D_{6} \mathrm{z}_{6}+D_{7} \mathrm{z}_{7}+D_{8} \mathrm{z}_{8},
\end{aligned}
$$

such that the following functional equality is satisfied:

$\left(y_{1}, y_{2}, y_{3}, y_{4}, y_{5}, y_{6}, y_{7}, y_{8}\right) \mapsto F\left(y_{1}, y_{2}, y_{3}, y_{4}, y_{5}, y_{6}, y_{7}, y_{8}\right)=$ 


$$
=M\left(y_{1}, y_{2}, y_{3}, \pi\left(y_{4}\right), \pi\left(y_{5}\right), \pi\left(y_{6}\right), \pi\left(y_{7}\right), \pi\left(y_{8}\right)\right),
$$

which, in turn, induces the following operator equations for statement functions from the constructions of mappings $F$ and $M$ :

$$
\begin{gathered}
A_{1}=D_{1}, A_{6}=D_{2}, B=D_{3}, \\
D_{1}=D_{4} \circ \pi, D_{2}=D_{5} \circ \pi, D_{3}=D_{6} \circ \pi, \\
D_{4}=D_{7} \circ \pi, \quad D_{5}=D_{8} \circ \pi .
\end{gathered}
$$

The Lemma 2 generalizes Corollary 1 [6].

Lemma 2. Let $S, Q \in \wp_{\mu}$, then $S \underset{\bmod \mu}{\subseteq}$ if the following equalities hold:

$S:=\{t \in T:(g(t), w(t), v(t), q(t), s(t), h(t), \hat{u}(t), \widetilde{u}(t))=0 \in U\}$, $Q:=\{t \in T: \dot{g}(t)=0 \in X\}, \dot{g}=d g d t(g, w, v, q, s, h, \hat{u}, \widetilde{u}) \in V_{N}$, $V_{N}:=\operatorname{Span}\{(d x d t, x, u, \pi(x, x), \pi(x, d x d t), \pi(d x d t, d x d t)$,

$\left.\pi(E(u), x), \pi(E(u), d x d d t)) \in \mathrm{L}_{2}(T, U):(x, u) \in N\right\}$.

Further, let $\mathrm{L}_{+}(T, R)$ be the convex cone $[4, \mathrm{p}$. 127] of the classes of $\mu$-equivalence of all real nonnegative functions $\mu$-measurable on $T$, and $S_{L}$ is such a quasi-ordering in $\mathrm{L}_{+}(T, R)$ that $\xi^{\prime} \leq_{\mathrm{L}} \xi^{\prime \prime}$ in case if $\xi^{\prime}(t) \leq \xi^{\prime \prime}(t) \mu$ is almost everywhere on the interval $T$. Moreover, for a given $W \subset \mathrm{L}_{+}(T, R)$, by $\sup _{L} W$ we denote the smallest upper face for $W$ if this face exists in the cone $\mathrm{L}_{+}(T, R)$ in the quasi-ordering structure $S_{L}$. In particular, the following occurs:

$\sup _{L}\left\{\xi^{\prime}, \xi^{\prime \prime}\right\}=\xi^{\prime} \vee \xi^{\prime \prime}:=2^{-1}\left(\xi^{\prime}+\xi^{\prime \prime}+\left|\xi^{\prime}-\xi^{\prime \prime}\right|\right)$.

In this formulation, we consider a functional lattice with orthocomplement [4, p. 339]:

$$
R(W):=\left\{\xi \in \mathrm{L}_{+}(T, R): \xi \leq_{\mathrm{L}} \sup _{\mathrm{L}} W\right\} .
$$

Then $\left(R(W), \leq_{L}\right)$ is the lattice with lower $\chi_{\varnothing} \in$ $\mathrm{L}_{+}(T, R)$ and upper $\sup _{\mathrm{L}} W \in \mathrm{L}_{+}(T, R)$ boundaries; here $\chi_{\varnothing}$ is the "null-function" of the convex cone $\mathrm{L}_{+}(T, R)$. Moreover, from Theorems 17 [3, $\mathrm{p}$. 68] and Corollary 1 [3, p. 69] it is easy to extract a more general statement; below $\inf _{L}$ is the largest lower $\leq_{\mathrm{L}}$-face.

Lemma 3. The functional lattice $R(W)$ is complete, i.e. we can perform inclusions

$$
\inf _{\mathrm{L}} V, \sup _{\mathrm{L}} V \in \mathbb{R}(W) \forall V \subseteq R(W) .
$$

Let $\Psi: V_{N} \rightarrow \mathrm{L}_{+}(T, R)$ be the Rayleigh-Ritz functional operator $[7,8]$ :

$$
t \mapsto \Psi(\varphi)(t):=\left\{\begin{array}{l}
\|\hat{A}(t) \dot{g}(t)\|_{X} /\|\varphi(t)\|_{U}, \text { if } \varphi(t) \neq 0 \in U ; \\
0 \in R, \text { if } \varphi(t)=0 \in U ;
\end{array}\right.
$$

where $\varphi:=(g, w, v, q, s, h, \hat{u}, \widetilde{u}) \in V_{N}$.

It is clear that the following equality occurs:

$$
\begin{aligned}
& \|\left.(g(t), w(t), v(t), q(t), s(t), h(t), \hat{u}(t), \widetilde{u}(t))\right|_{U}:= \\
& =\left(\|g(t)\|_{X}^{2}+\|w(t)\|_{X}^{2}+\|v(t)\|_{Y}^{2}+\|q(t)\|_{Z}^{2}+\right. \\
& \left.+\|s(t)\|_{Z}^{2}+\|h(t)\|_{Z}^{2}+\|\hat{u}(t)\|_{Z}^{2}+\|\left.\widetilde{u}(t)\right|_{Z} ^{2}\right)^{1 / 2} .
\end{aligned}
$$

By virtue of Lemma 2, in the time interval $T$ the following equality holds:

$$
\operatorname{supp} \Psi(\varphi) \underset{\bmod \mu}{=} \operatorname{supp}\|\hat{A} \dot{g}\|_{X} ;
$$

In the definition of the supp-structure of the function support, we follow [3, p. 137] (that is, the supp structure of the function support is determined within the accuracy of a set of measure zero; not to be confused with the supp -support from [18]).

Nonlinear operator (2) satisfies very simple (but important) relations

$$
\chi_{\varnothing} \bigcirc_{L} \Psi(\varphi)=\Psi(r \varphi), r \in R^{*}:=R \backslash\{0\}, \varphi \in V_{N} ;
$$

in the designations below we will distinguish the image of the point $\Psi(\varphi)$ and the image of the set $\Psi[\{\}\}$.

The theory of the Rayleigh-Ritz operator needs a precise functional-geometric language that makes us pay special attention to this language. So before we go any further, let us introduce additional terminology. Namely, in view of (3), the operator $\Psi$ induces the mapping

$$
P \Psi: P_{N} \rightarrow \mathrm{L}_{+}(T, R),
$$

which will be called the projectivization of the Rayleigh-Ritz operator, and defined as

$$
P \Psi(\gamma):=\Psi[\gamma], \gamma \in P_{N}\left(\gamma \subset V_{N}\right),
$$

where $P_{N}$ is the real projective space associated with linear variety $V_{N}$ (with topology induced from space $\left.\mathrm{L}_{2}(T, U)\right)$; , i.e. $P_{N}$ is a set of orbits of the multiplicative group $R^{*}$, acting on $V_{N} \backslash\{0\}$. In this geometric interpretation, the key moment is topological properties of space $P_{N}, \operatorname{dim} P_{N}<\aleph_{0}$, of course, in the first place (within the context of Theorem 2), its compacted-ness. Specifically, if $\operatorname{dim} V_{N}=3$, then the compact 2-manifold $P_{N}$ is arranged as the Möbius loop, to which a circle is glued along its border [13, p. 162]. As a side note, we can state that on $P_{N}$ one can introduce the structure of the $C W$-complex, which, in turn, simplifies the consideration of the issue of geometric implementation (Theorem 9.7 [13, p. 149]) of the manifold $P_{N}$.

Theorem 1. Each of the following three conditions results in the other two:

(i) BDR-problem (1) is solvable with respect to statement functions

$$
\left(A, A_{,}, B, \mathrm{D}_{1}, \mathrm{D}_{2}, \mathrm{D}_{3}, \mathrm{D}_{4}, \mathrm{D}_{5}\right) \in \mathrm{L}_{2} ;
$$


(i) $\exists \theta \in \mathrm{L}_{2}(T, R): \Psi(\varphi) \leq_{\mathrm{L}} \theta \forall \varphi \in V_{N}$;

(iii) $\exists \sup _{\mathrm{L}} P \Psi\left[P_{N}\right]$ : $\sup _{\mathrm{L}} P \Psi\left[P_{N}\right] \in \mathrm{L}_{2}(T, R)$.

At the same time, to perform the inclusion

$$
\left(A, A_{0}, B, D_{1}, D_{2}, D_{3}, D_{4}, D_{5}\right) \in L^{*}
$$

it is necessary to

$$
R\left(P \Psi\left[P_{N}\right]\right) \subset \mathrm{L}_{\infty}(T, R) .
$$

Remark 1. Theorem 1 can be seen as an initial step in the study of the problem, when the bundle of controllable trajectory curves $N$, induced from the implicit differential equation of the higher order, is required to compare the explicit unsteady bilinear differential system of the second order that contains the same bundle of trajectory curves $N$.

Proof. We will use the ideas of work [14]. Adhering to Definition 1 [14], we will introduce into consideration the structure of $M_{2}$-operator

$$
M: \mathrm{L}_{2}(T, U) \rightarrow \mathrm{L}_{1}(T, X)
$$

of the form

$$
\begin{gathered}
\exists\left(D_{1}, D_{2}, D_{3}, D_{4}, D_{5}, D_{6}, D_{7}, D_{8}\right) \in \mathbf{I}_{2}: \\
M(g, w, v, q, s, h, \hat{u}, \widetilde{u}):= \\
=D_{1} g+D_{2} w+D_{3} v+D_{4} q+D_{5} s+D_{6} h+D_{3} \hat{u}+D_{8} \widetilde{u} \\
\forall(g, w, v, q, s, h, \hat{u}, \widetilde{u}) \in \mathrm{L}_{2}(T, U) .
\end{gathered}
$$

The rest of the proof details with minor clarifications (taking into account Lemma 1, Lemma 2 and Lemma 3 for the lattice $\left.R\left(P \Psi\left[P_{N}\right]\right)\right)$ is included into an analytical diagram of the $M_{2}$-continuability in the form of Corollary 2 and Theorem 3 [14]. At this, the necessary condition for the

$$
\left(A, A_{0}, B, D_{1}, D_{2}, D_{3}, D_{4}, D_{5}\right) \in \mathbb{L}^{*}
$$

is set up by modifying the proof of Theorem 3 [7].

Remark 2. Even in case of $1<\operatorname{Card} N<\aleph_{0}$, $\operatorname{Card} P_{N}=\exp \aleph_{0}$ occurs; but one can show that there exists (Theorem 17, [3, p. 68]) a countable set $G \subset P_{N}$ such that if in $\mathrm{L}_{+}(T, R)$ lies $\sup _{\mathrm{L}} P \Psi\left[P_{N}\right]$, then the function $\zeta:=\sup _{\mathrm{L}} P \Psi\left[P_{N}\right]$ is executed by the following sup-structure:

$$
t \mapsto \zeta(t)=\sup \{P \Psi(\gamma)(t) \in R: \gamma \in G\} .
$$

Remark 3. Relying on Theorem 1 , it is not difficult to formulate an analogue of Theorem 3 [12], expressing in terms of angular distance (in the Hilbert space) the geometric conditions of the existence of the bilinear system (1), implementing dynamic bundles $N_{1}, N_{2}$, each of which has its BDR-model, and specifically in the formulation of work [7], when simulated operators of the differential system (1) are stationary, i.e.

$$
\left(A, A_{f}, B, D_{1}, D_{2}, D_{3}, D_{4}, D_{5}\right) \in \mathbb{L}^{*},
$$

among other things, with the optimal operator norm.

The following particular case is also important in specific discussions:

Corollary 1. If $\operatorname{dim} V_{N}<\aleph_{0}, \Psi\left[V_{N}\right] \subset \mathrm{L}_{2}(T, R)$ and there is a $p \in[1, \infty)$, at which

$$
\begin{gathered}
\Psi\left(\varphi_{1}+\varphi_{2}\right) \leq_{\mathrm{L}} p \Psi\left(\varphi_{1}\right)+p \Psi\left(\varphi_{2}\right), \\
\left(\varphi_{1}, \varphi_{2}\right) \in V_{N} \times V_{N},
\end{gathered}
$$

then the BDR-problem (1) is solvable.

Note, that at $p=1$, this property (in the context of quasi-ordering of $\leq_{L}$ ) is akin to the property of "sublinearity" [18. p. 400] of functional operators.

\section{The Continuity Property of the Rayleigh-Ritz Operator}

In the case of compactness of the projective manifold $P_{N}\left(\operatorname{dim} P_{N}<\mathcal{S}_{0}\right.$ is of equal value) it is natural to try linking this geometric property to the problem of constructing the lattice $R\left(P \Psi\left[P_{N}\right]\right)$ within the context of the Rayleigh-Ritz operator projectivization continuity conditions; below in Theorem 2, when selecting metric structure in the cone $\mathrm{L}_{+}(T, R)$, we resorted to Theorem 15, 16 [3, pp. 65, 67]; in this formulation, the convex cone $\mathrm{L}_{+}(T, R)$ forms a complete separable metric space.

Theorem 2. Let $\operatorname{dim} P_{N}<\aleph_{0}$ and the convex cone $\mathrm{L}_{+}(T, R)$ be endowed with a topology induced by convergence in measure $\mu$, or, equivalently, by the invariant metric

$$
\begin{gathered}
\rho_{T}(\cdot,): \mathrm{L}_{+}(T, R) \times \mathrm{L}_{+}(T, R) \rightarrow R, \\
\rho_{T}\left(f_{1}, f_{2}\right):=\int_{T}\left|f_{1}(\tau)-f_{2}(\tau)\right|\left(1+\left|f_{1}(\tau)-f_{2}(\tau)\right|\right)^{-1} \mu(d) . .
\end{gathered}
$$

Then the operator

$$
P \Psi: P_{N} \rightarrow \mathrm{L}_{+}(T, R)
$$

will be continuous if the dynamic bundle $N$ is such that the following takes place:

$$
\forall \varphi \in V_{N} \backslash\{0\}: \operatorname{supp}\|\varphi\|_{U} \underset{\bmod \mu}{=} T,
$$

in particular, if

$$
\forall \gamma \in P_{N}: \operatorname{supp} P \Psi(\gamma) \underset{\bmod \mu}{=} T .
$$

It should be noted that Theorem 2 is the development of Theorem 3, which confirms its methodological importance in mathematical (a posteriori) modeling of complex dynamic systems. The first application of this result is the following statement.

Corollary2. If, when performing (4) or (5), the operator $P \Psi$ is one-to-one, then $P \Psi$ is homeomorphism, and the fundamental group of metric 
space $\left(P \Psi\left[P_{N}\right], \rho_{T}\right)$ is isomorphic to the additive group of whole numbers $Z$ at $\operatorname{dim} \operatorname{Span} N=2$, and the group of deductions $Z_{2}$, at $\operatorname{dim} \operatorname{Span} N \geq 3$. Moreover, space $\left(P \Psi\left[P_{N}\right], \rho_{T}\right)$ is orientable if the dimensionality of the linear shell Span $N$ is even, and is non-orientable if this dimensionality is odd.

Proof. The homeomorphism of the operator $P \Psi$ follows from Theorem 3.1.13 [19, p. 199]. This allows one to calculate (by virtue of Theorem 2.3 $[13, \mathrm{p} .47]$ and proof of the Theorem 12.1 [13, p. $174]$ the fundamental group of space $\left(P \Psi\left[P_{N}\right], \rho_{T}\right)$; as homeomorphic spaces are the spaces of the same homotopic type.

Corollary 3. The ultimate projectivization

$$
P \Psi: P_{N} \rightarrow \mathrm{L}_{+}(T, R)
$$

is continuous if for the arbitrary function $\varphi \in V_{N} \backslash\{0\}$ and any $t \in T_{\varphi}:=\{t \in T: \varphi(t)=0 \in U\}$ there is a number $\delta_{\varphi t}>0$ such that $\mu\left(\left(t-\delta_{\varphi t}, t+\delta_{\varphi t}\right) \cap T_{\varphi}\right)=0$. At the same time, $P \Psi\left[P_{N}\right]=f[F]$, where $F \subset[0,1] \subset R$ is the Cantor set, $f: F \rightarrow \mathrm{L}_{+}(T, R)$ is some continuous mapping.

Proof. We will establish the fact of $\mu\left(T_{\varphi}\right)=0$ (which is equivalent to (4)). To do this, we will select the number $\delta_{t}>0$ to each moment $t \in T_{\varphi}$ so that

$$
\mu\left(\left(\mathrm{t}-\delta_{t}, t+\delta_{t}\right) \cap T_{\varphi}\right)=0 .
$$

Next, we'll find such rational numbers $r_{t-}, r_{t+}$, that $r_{t-} \in\left(t-\delta_{t}, t\right), r_{t+} \in\left(t, t+\delta_{t}\right)$, and let $I_{t}:=\left(r_{t-}, r_{t+}\right)$. Then the family of intervals $\left\{I_{t}\right\}_{t \in T_{\varphi}}$ covers the set $T_{\varphi}$, and since each interval of $I_{t}$ is open with rational end points, the family $\left\{I_{t}\right\}_{t \in T_{\varphi}}$ contains a certain countable subfamily of $\left\{I_{t_{i}}\right\}_{i=1,2, \ldots}$, which is also the cover of the set $T_{\varphi}$.

Since for any index $i=1,2, \ldots$ the inclusion of $I_{t_{i}} \subset\left(t_{i}-\delta_{t_{i}}, t_{i}+\delta_{t_{i}}\right)$, is made, then, evidently, the equality $\mu\left(I_{t_{i}} \cap T_{\varphi}\right)=0$ occurs, and it means that the chain of $\mu$-relations is fair:

$$
\begin{aligned}
\mu\left(T_{\varphi}\right) & =\mu\left(T_{\varphi} \cap\left(\cup_{i=1,2, . .} I_{t_{i}}\right)\right)=\mu\left(\cup_{i=1,2, .}, T_{\varphi} \cap I_{t_{i}}\right) \leq \\
& \leq \sum_{i=1,2, .} \mu\left(T_{\varphi} \cap I_{t_{i}}\right)=0 \Rightarrow \mu\left(T_{\varphi}\right)=0 .
\end{aligned}
$$

Since the compact manifold $P_{N}$ is locally arranged as a finite-dimensional Euclidean space, the equality of $P \Psi\left[P_{N}\right]=f[F]$ can be determined with Theorem 4.11 [13, p. 77] and Theorem 9.7 [13, p. 97].

Taking into account that the continuous real function in the compact space reaches its greatest and lowest values, we come to the conclusion that in the formulation of Corollary 3 and Theorem 3.1.10 [19,p. 199], for the case when $1 \leq \operatorname{dim}_{N}<\aleph_{0}$ and with $\sup _{\mathrm{L}} P \Psi\left[P_{N}\right]$, there will be such points $\gamma^{\prime}, \gamma^{\prime \prime} \in P_{N}$ that the following is fulfilled:

$$
\begin{gathered}
\rho_{T}\left(P \Psi\left(\gamma^{\prime}\right), \chi_{\varnothing}\right)=\sup \left\{\rho_{T}\left(P \Psi(\gamma), \chi_{\varnothing}\right): \gamma \in P_{N}\right\}< \\
<\rho_{T}\left(\sup P \Psi\left[P_{N}\right], \chi_{\varnothing}\right)<\mu(T), \\
\rho_{T}\left(P \Psi\left(\gamma^{\prime \prime}\right), \sup _{\mathrm{L}} P \Psi\left[P_{N}\right]\right)= \\
=\inf \left\{\rho_{T}\left(P \Psi(\gamma), \sup _{\mathrm{L}} P \Psi\left[P_{N}\right]\right): \gamma \in P_{N}\right\} \neq 0 .
\end{gathered}
$$

It should be noted that the inclusion of $P \Psi\left(\gamma^{\prime}\right) \in$ $\mathrm{L}_{2}(T, R)$ does not guarantee an embedding

$$
R\left(P \Psi\left[P_{N}\right]\right) \subset \mathrm{L}_{2}(T, R) .
$$

At the same time, it should be noted that the condition $\operatorname{dim} P_{N}=0$ leads to an important proposition

$$
\begin{gathered}
\sup _{\mathrm{L}} P \Psi\left[P_{N}\right]=P \Psi\left[P_{N}\right]= \\
=\left\|\hat{A} d^{2} x / d t^{2}\right\|_{X} /\left(\left\|d x / d t_{X}^{2}+\right\| x\left\|_{X}^{2}+\right\| u\left\|_{Y}^{2}+\right\| x \|_{X}^{4}+\right. \\
\left.+\left\|\left.x\right|_{X} ^{2}\right\| d x d t_{X}^{2}+\left\|d x d d t_{X}^{4}+\right\| E(u)\left\|_{X}^{2}\right\| x\left\|_{X}^{2}+\right\| E(u)\left\|_{X}^{2}\right\| d x d d t_{X}^{2}\right)^{1 / 2} .
\end{gathered}
$$

Within the context of Theorem 1, 2, one can clarify the conditions of the existence of the lattice $R\left(P \Psi\left[P_{N}\right]\right)$. As a starting point, we introduce an auxiliary topological construction, namely, for the natural $n$ let $W_{n}$ denote some finite $n^{-1}$-dense [19, p. 395] subset in metric space $\left(P \Psi\left[P_{N}\right], \rho_{T}\right)$.The subset $W_{n}$ is found in the virtue of Theorem 2 and Theorem 4.3.27 [19, p. 408]. Below, $\operatorname{Lim}_{\rho_{T}}\left\{\xi_{n}\right\}$ means the limit of sequence $\left\{\xi_{n}\right\} \subset \mathrm{L}_{+}(T, R)$ in the topology induced by the metric $\rho_{T}$.

\section{Corollary 4. Let}

$$
\begin{gathered}
\left\{W_{i}\right\}_{i=1, \ldots, n}, W_{i}=\left\{\zeta_{1}, \ldots, \zeta_{k_{i}}\right\} \subset P \Psi\left[P_{N}\right], \\
f_{n}:=\xi_{1} \vee \ldots \vee \xi_{n}, \xi_{i}=\zeta_{1} \vee \ldots \vee \zeta_{k_{i}}, 1 \leq i \leq n .
\end{gathered}
$$

Then the cone $\mathrm{L}_{+}(T, R)$ contains the lattice $\mathcal{R}\left(P \Psi\left[P_{N}\right]\right)$ if and only if $\rho_{T}\left(f_{n}, f_{m}\right) \rightarrow 0(n, m \rightarrow \infty)$, besides, the BDR-solvability takes the following form: the pair $(N, \hat{A})$ has a differential realization (1) then and only then, when $\operatorname{Lim}_{p_{T}}\left\{f_{n}\right\} \in \mathrm{L}_{2}(T, R)$, which is equivalent to $R\left(P \Psi\left[P_{N}\right]\right) \subset \mathrm{L}_{2}(T, R)$.

In concluding, here are the examples illustrating (in some essential ways) the possible analytical approach in the functional analysis of the pair $(N, \hat{A})$, leading to the use of Theorems 1, 2 .

Example 1. Let

$\mathrm{L}_{\times}(T, R):=\left\{(f, g) \in \mathrm{L}_{+}(T, R) \times \mathrm{L}_{+}(T, R): \operatorname{supp} f \underset{\bmod }{\subseteq \operatorname{supp}}\right\}^{\prime} ;$ 
$\mathrm{L}_{\times}(T, R)$ is not a linear space, but it contains them (including infinite-dimensional ones).

Let us take a look at the functional operator

$$
\psi: \mathrm{L}_{\times}(T, R) \rightarrow \mathrm{L}_{+}(T, R)
$$

with construction of the form:

$$
\begin{gathered}
\psi(f, g)(t):=f(t) g^{-1}(t) \text { at } g(t) \neq 0, \\
\psi(f, g)(t):=0 \text { for } g(t)=0 ;
\end{gathered}
$$

With Lemma 2 in mind, this structure is induced by the operator (2).

Next, let us introduce the sequence $\left\{\left(f_{n}, g_{n}\right)\right\} \subset$ $\mathrm{L}_{\times}(T, R)$, for which the following holds true:

$$
\rho_{T}\left(f_{n}, f_{m}\right) \rightarrow 0, \rho_{T}\left(g_{n}, g_{m}\right) \rightarrow 0(n, m \rightarrow \infty) .
$$

Moreover, taking into account the completeness of the metric space $\left(\mathrm{L}_{+}(T, R), \rho_{T}\right)$, we will require that

$$
\left(\operatorname{Lim}_{\rho_{T}}\left\{f_{n}\right\}, \operatorname{Lim}_{\rho_{T}}\left\{g_{n}\right\}\right) \in \mathrm{L}_{\times}(T, R) ;
$$

for convenience, let us denote

$$
f:=\operatorname{Lim}_{\mathrm{P}_{T}}\left\{f_{n}\right\}, g:=\operatorname{Lim}_{\mathrm{P}_{T}}\left\{g_{n}\right\} .
$$

In this formulation, for convergence

$$
\rho_{T}\left(\psi\left(f_{n}, g_{n}\right), \psi(f, g)\right) \rightarrow 0(n \rightarrow \infty)
$$

it is enough to

$$
\lim \left\{\mu\left(\operatorname{supp} g_{n} \Delta \operatorname{supp} g\right): n \rightarrow \infty\right\}=0,
$$

where $\Delta$ is the symmetric difference of $\operatorname{supp} g_{n}$ and suppg, i.e. ( $\left.\operatorname{supp} g_{n} \backslash \operatorname{supp} g\right) \cup\left(\operatorname{supp} g \backslash \operatorname{supp} g_{n}\right)$.

Although condition (7) is interesting and useful [20], unfortunately, it is not necessary ${ }^{2}$, which indicates that there is a certain "excess" of conditions (4), (5) of Theorem 2. Within this context, there is an open issue of constructing a characteristic condition for the pair $\left(\left\{\left(f_{n}, g_{n}\right)\right\},(f, g)\right)$ that defines the $\rho_{T}$-convergency of the following form:

$$
\rho_{T}\left(\psi\left(f_{n}, g_{n}\right), \psi(f, g)\right) \rightarrow 0(n \rightarrow \infty) .
$$

Example 2. Let $T=[0,10], Y:=X, \hat{A}$ is the identical operator (homothetic transformation with coefficient 1 [18, p. 87]), $e \in X,\|e\|_{X}=1$ and

$$
\begin{gathered}
A_{1}=0 \in L(X, X), \\
\mathrm{D}_{1}=\mathrm{D}_{3}=\mathrm{D}_{4}=\mathrm{D}_{5}=0 \in \mathcal{L}\left(X^{2}, X\right), \\
t \mapsto x(t)=(t \sin t) e, t \mapsto u(t)=0 \in \mathrm{L}_{2}(T, X) .
\end{gathered}
$$

\footnotetext{
${ }^{2}$ The condition (7) becomes necessary to perform the convergence

$$
\rho_{T}\left(\psi\left(f_{n}, g_{n}\right), \psi(f, g)\right) \rightarrow 0(n \rightarrow \infty),
$$

if to overload the sequence $\left\{f_{n}\right\}$ by the additional proposition: there is such $\delta>0$ for which $f_{n}(t) \geq \delta$ for any $n$ and $\mu$ of almost all $t \in \operatorname{suppg}$ (see Remark 2 [20]).
}

Then (see Fig. 1$)^{3}$ the function

$$
\begin{gathered}
f:=\sup _{\mathrm{L}} P \Psi\left(P_{N}\right)= \\
\left\|d^{2} x / d t^{2}\right\|_{X}\left(\|x\|_{X}^{2}+\|x\|_{X}^{2}\|d x d t\|_{X}^{2}\right)^{-1 / 2} ;
\end{gathered}
$$

does not belong to the space $\mathrm{L}_{2}(T, R)$ ), therefore, according to Theorem 1 and formula (6), realization (1) for the uncontrolled process $t \mapsto(x(t), u(t))$ does not exist.

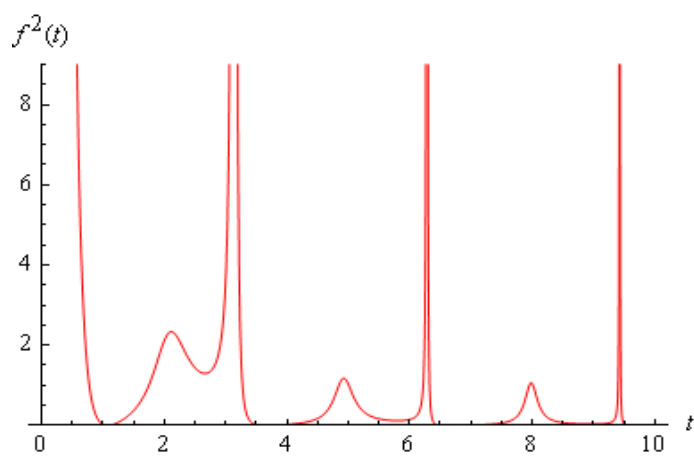

Fig. 1. $f^{2}(t)=(2 \cos t-t \sin t)^{2} \times$

$\times\left((t \sin t)^{2}+(t \sin t)^{2}(\sin t+t \cos t)^{2}\right)^{-1}$

Example 3. Let us change (see Fig. 2) the formulation of Example 2 by the fact that $t \mapsto u(t)=\left(t \sin ^{2} t+2^{-1} t^{2} \sin 2 t+\cos t\right) e$.

Then, obviously, we have

$$
f:=\sup _{\mathrm{L}} P \Psi\left(P_{N}\right)=
$$

$=\mid d^{2} x / d\left\|^{2}\right\|_{X}\left(\|x\|_{X}^{2}+\left.\left\|\left.x\right|_{X} ^{2}\right\| d x d t\right|_{X} ^{2}+\|\left. u\right|_{Y} ^{2}\right)^{-1 / 2} \in \mathrm{L}_{2}(T, R)$, and, according to Theorem 1, differential realization (1) for the controlled dynamic process $t \mapsto(x(t), u(t))$ exists; it is easy to establish that the realization has the form of:

$$
d^{2} x / d t^{2}+x=2 u-2 \mathrm{D}_{2}(x, d x / d t)
$$
$\mathrm{D}_{2}=\langle\because\rangle_{X} e,\langle\because\rangle_{X}$ is the scalar product in $X$.

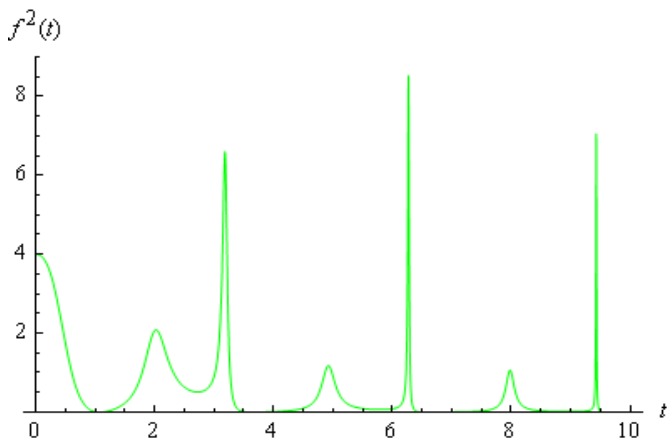

Fig. 2. $f^{2}(t)=(2 \cos t-t \sin t)^{2} \times\left((t \sin t)^{2}+\right.$ $\left.+(t \sin t)^{2}(\sin t+t \cos t)^{2}+\left(t \sin ^{2} t+2^{-1} t^{2} \sin 2 t+\cos t\right)^{2}\right)^{-1}$

${ }^{3}$ The symbolic calculations of the function $f^{2}(\cdot)$ can be carried out (by computer algebra) using a special software environment [21], adapted to automate the problem-solving of analytical mechanics. 
Example 4. Let us assume that $N=\{(x, u)\}$ and $\hat{A}_{1}, \hat{A}_{2} \in \mathrm{L}_{\infty}(T, L(X, X))$ are set on the interval $T=[0,1]$, and also for $\left(N, \hat{A}_{1}, \hat{A}_{2}\right)$ there will be such $\theta_{1}, \theta_{2}, \theta_{3}, \theta_{4} \in \mathrm{L}_{\infty}(T, R)$ that

$$
\begin{aligned}
t \mapsto\|x(t)\|_{X}=t \theta_{1}(t), \quad t \mapsto\|d x(t) / d t\|_{X}=t \theta_{2}(t), & t \mapsto\|u(t)\|_{Y}=t \theta_{3}(t), \\
t & \mapsto\left\|\hat{A}_{1}(t) d^{2} x(t) / d t^{2}\right\|_{X}=t \theta_{4}(t), \\
t & \mapsto\left\|\hat{A}_{2}(t) d^{2} x(t) / d t^{2}\right\|_{X}=\theta_{4}(t),
\end{aligned}
$$

with that, there are real numbers $\delta, \varepsilon>0$, for which

$$
\begin{gathered}
\mu\left\{t \in T: \theta_{1}(t)+\theta_{2}(t)+\theta_{3}(t)<\delta\right\}= \\
=\mu\left\{t \in T: \theta_{4}(t)<\varepsilon\right\}=0 .
\end{gathered}
$$

Then it is not difficult to establish (by virtue of Theorem 1, as well as by functional relations (6)), that the problem of BDR-model is solvable for the pair $(N, \hat{A})$ and is not solvable for the pair $(N, \hat{A})$.

\section{Conclusion}

A qualitative theory of non-linear non-stationary differential realization, considered in the spirit of the infinite-dimensional proposition of the reverse problems of mathematical physics, is more complicated, interesting, deeper in the applications and is very important for understanding the basic properties of the differential models themselves. Its geometric structures can serve as the starting points for the modern development of the general (axiomatic) theory of systems (in keeping with Chapter III [22]), simultaneously creating a reputation for these structures as a useful mathematical tool in the precision a posteriori modeling of complex dynamic models.

Since in many practically important objectives of realizing the differential representation of the simulated dynamic processes it is necessary to take into account the nonlinear relationship both from the trajectory itself and the speed of motion on it, and from program control, then the main attention was focused on the study of the realization model, which depends on five non-stationary bilinear structures. Moreover, one of them is set on the trajectory itself, the second bilinear operator depends on the trajectory and the speed of motion on it, the third bilinear operator depends only on the speed of motion along this trajectory, and the other two take these variables into account in connection with the program controlling influencing them.

On the other hand, the goal of the article was also, without going into numerous clarifying details and avoiding the morass of mathematical generalizations, to advance in studying the qualitative geometric properties of a non-stationary second-order BDRmodel as far as possible without involving a complex topological-algebraic technique.

Even now it is possible to quite confidently indicate system-theoretical direction, which will form the basis of the next stage in the development of a qualitative theory of the implementation of higher orders, namely, transition from the bilinear structure of non-linear bonds to the multi-linear structure. Methodologically, this transition lies in the plane of use of the language of tensor structures of Fock spaces $[4$, p. 68$]$ and projective ideas $[13,16]$. This language in this statement of nonlinear precision differential modeling of the dynamics of controlled infinite-dimensional continuous behavioristic systems should be given credit. On the one hand, it is compact and relatively flexible, and on the other hand, it is in its analytical constructions that the geometrical intuition is developed during the analysis of infinite-dimensional vector fields, which is one of the main driving forces for further synthetic development of the general theory of nonlinear differential realization. Such qualitative investigations suggest a deep penetration into the physical content of the subject [1], guided by the idea that the subject of non-stationary multilinear differential realization comes from the apodictic simplicity of some higher kind, at least if you adhere to Popper's point of view ${ }^{4}$.

Acknowledgments: This work was supported by the Russian Foundation for Basic Research (Projects: No. 19-01-00301, No. 19-08-00746). Some other helpful studies can be found in as [24] and [25].

\section{References}

[1] J.C. Willems, System theoretic models for the analysis of physical systems, Ric. Aut., Vol. 10, No. 1979, pp. 71-106.

[2] N.U. Ahmed, Optimization and Identification of Systems Governed by Evolution Equations on Banach Space, John Wiley and Sons, New York, 1988.

[3] L.V. Kantorovich and G.P. Akilov, Functional Analysis, Nauka, Moscow, 1977 (in Russian).

[4] M. Reed and B. Simon, Methods of Modern Mathematical Physics 1. Functional Analysis, Academic Press, New York, 1972; translated under Mir, Moscow, 1977.

[5] Yu.L. Daletsky and S.V. Fomin, Measures andDifferential Equations on Infinitely-Dimensional Spaces, Nauka, Moscow, 1983 (in Russian).

${ }^{4}$ K.R. Popper (a logician, a representative of analytical philosophy) put forward the principle of falsification (refutability), according to which the criterion of the scientific nature of a theory is determined by the possibility of its refutation by experience [23, p. 36]. 
[6] V.A. Rusanov, L.V. Antonova and A.V. Daneev, Inverse problem of non-linear systems analysis: A behavioral approach, Advances in Differential Equations and Control Processes, Vol. 10, No. 2, 2012, pp. 69-88.

[7] V.A. Rusanov, A.V. Daneev, A.V. Lakeev and Yu.É. Linke, On the differential realization theory of nonlinear dynamic processes in a Hilbert space, Far East Journal of Mathematical Sciences, Vol. 97, No. 4, 2015, pp. 495-532.

[8] V.A. Rusanov, A.V. Daneev and Yu.É. Linke, To the geometrical theory of the differential realization of dynamic processes in a Hilbert space, Cybernetics and Systems Analysis, Vol. 53, No. 4, 2017, pp. 554-564.

[9] V.A. Rusanov, A.V. Daneev, A.V. Lakeyev and V.N. Sizykh, Higher-order differential realization of polylinear-controlled dynamic processes in a Hilbert space, Advances in Differential Equations and Control Processes, Vol. 19, No. 3, 2018, pp. 263-274.

[10] A.V. Daneev, V.A. Rusanov and M.V. Rusanov, From Kalman-Mesarovic realization to a normal-hyperbolic linear model, Cybernetics and Systems Analysis, Vol. 41, No. 6, 2005, pp. 909-923.

[11] V.A. Rusanov, A.V. Banshchikov, A.V. Daneev and A.V. Lakeyev, Maximum entropy principle in the differential second-order realization of a nonstationary bilinear system, Advances in Differential Equations and Control Processes, Vol. 20, No. 2, 2019, pp. 223-248.

[12] A.V. Lakeev, Yu.É. Linke and V.A. Rusanov, Realization of a polylinear controller as a second-order differential system in a Hilbert space, Differential Equations, Vol. 53, No. 8, 2017, pp. 1070-1081.

[13] V.V. Prasolov, Elements of Combinatorial and Differentiable Topology, MTsNMO, Moscow, 2014 (in Russian).

[14] V.A. Rusanov, A.V. Lakeev and Yu.É. Linke, Existence of a differential realization of a dynamical system in a Banach space in the constructions of extensions to $M_{p}$-operators, Differential Equations, Vol. 49. No. 3, 2013, pp. 346-358.

[15] V.A. Rusanov, A.V. Daneev, A.V. Lakeev, Yu.E. Linke and A.A. Vetrov, System-theoretical foundation for identification of dynamic systems. II, Far East Journal of Mathematical Sciences, Vol. 116, No. 1, 2019, pp. 25-68.

[16] A.A. Kirillov, Elements of Representation Theory, Nauka, Moscow, 1978 (in Russian).

[17] R.E. Kalman, P.L. Falb and M.A. Arbib, Topics in Mathematical System Theory, MC GrawHill Book Company, New York, 1969; translated under Mir, Moscow, 1971.

[18] R.E. Edwards, Functional Analysis: Theory and Applications, Holt, New York,1965; translated under Mir, Moscow, 1969.

[19] R. Engelking, General Topology, PWN, Warszawa, 1977; translated under Mir, Moscow, 1986.

[20] A.V. Lakeyev, Yu.E. Linke and V.A. Rusanov, On a criterion for continuity of the Rayleigh-Ritz operator, Vestnik Buryat. Gos. Univ. Mat. i Informat, No. 3, 2018, pp. 3-13 (in Russian).

[21] A.V. Banshchikov and L.A. Bourlakova, Computer algebra and problems of motion stability, Mathematics and Computer in Simulation, Vol. 57, No. 3, 2001, pp. 161-174.

[22] M.D. Mesarovic and Y. Takahara, General Systems Theory: Mathematical Foundations, Academic Press, New York, 1975; translated under Mir, Moscow, 1978.

[23] K.R. Popper, Conjecture and Refutations, Harper and Row, London, 1963.

[24] Dzenan Gusic, On Some Higher Order Counting Functions for PSL $(2, R)$, WSEAS Transactions on Systems and Control, Volume 15, 2020, pp. $73-80$

[25] Dzenan Gusic, On the Error Terms of Chebyshev Functions for SL4 WSEAS Transactions on Systems and Control, Volume 15, 2020, pp. 57-63 